\title{
Anosmia in adolescence: what to do?
}

\begin{abstract}
Olfaction disorders are a rare condition in children whose diagnosis can be difficult due their inability to describe their perceptions correctly, delaying the diagnosis until adolescence. Structural or inflammatory diseases, isolated olfactory bulbs malformations or in the context of several syndromes, such as Kallmann's, are among the etiologies to be considered. The olfactometry and the Magnetic Resonance Imaging (MRI) are the main diagnostic tools. We report an exceptional case of an isolated agenesis of the olfactory bulbs in a 15years old girl.
\end{abstract}

Keywords: Anosmia; Adolescence; Agenesis; Olfactory bulbs
Volume 9 Issue I - 2017

\author{
Rijo Cedeño J, Pinilla Urraca M,Arellano B, \\ Fernandez Manzano C, Carbonell S \\ Department of Otorhinolaryngology Head Neck Surgery \\ University Hospital, Spain
}

\begin{abstract}
Correspondence: Jefferson Rijo Cedeño, Department of Otorhinolaryngology Head \& Neck Surgery, Puerta de HierroMajadahonda, University Hospital, Universidad Autónoma Madrid, Spain, Tel +34-663421991, Email jeff.rc26@gmail.com
\end{abstract}

Received: August 16,2017 | Published: November 06, 2017
Abbreviations: MRI, magnetic resonance imaging; UPSIT, university of pennsylvania smell identification test; ENT, ear nose and throat

\section{Introduction}

Diagnosing an olfactory dysfunction in small children can be a real challenge for the pediatricians and/or otolaryngologists, especially congenital alterations, as it is a subjective perception not well described by a kid. These alterations are normally described by the parents as a suspicion, until the children are old enough to complaint by themselves. Olfactory dysfunctions are in frequently found in children and adolescents. The most common causes of hyposmia in this period of life are disorders such as adenoids hypertrophy, nasal septum deviation, hypertrophy of middle and/or inferior turbinate and juvenile angiofibroma. Some other exceptional causes are brain tumors, endocrine disorders such as thyroid dysfunctions or Addison's disease, craniofacial trauma, autoimmune and degenerative diseases or any olfactory impairment caused by chemotherapy or any other drug. ${ }^{1,2}$ The agenesis of the olfactory nerve is an extremely rare entity that causes congenital anosmia and it usually appears in combination with other neurological malformations. It is more often associated with a hypogonadotropic hypogonadism in the so called Kallmann Syndrome with an incidence of 1 in 10,000 men and 1 in 50,000 women. ${ }^{1-3}$ The existence of anosmia in a child or adolescent requires a thorough investigation of the possible diseases that might be causing it. We report a case of a 15years old girl with an agenesis of the olfactory bulbs and a hypoplasia of the olfactory tracts with no relation to any syndrome or any other middle line malformation.

\section{Case report}

A 15years old girl was referred to the ENT Department of our hospital with a history of a lifelong impossibility to perceive smells She also complained of abundant rhinorrhea and occasional headaches. She didn't have any relevant family history and had a normal physical and psychological development. Rhinoscopy and fiberoscopy showed no structural abnormalities, no septal deviation, polyps nor any other inflammatory findings that could justify the symptoms. The olfactometry using the University of Pennsylvania Smell identification Test (UPSIT) described a total anosmia. Magnetic Resonance Imaging (MRI) found the absence of olfactory bulbs and a hypoplasia of both olfactory sulcus, more severe on the left one (Figure 1). Due to these findings, were referred our patient to the Endocrinology and Gynecology Departments of our hospital, where they determined a normal hormonal and sexual development. Thus the diagnosis of isolated congenital agenesis of the olfactory bulbs and hypoplasia of olfactory sulcus was made.

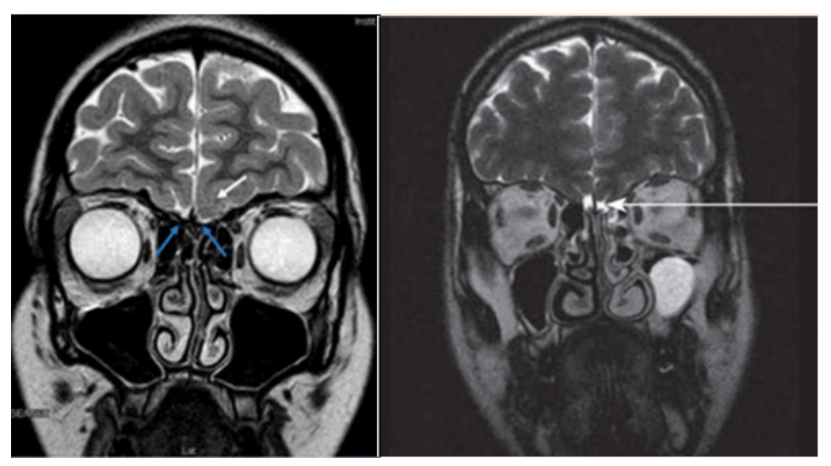

Figure I Coronal section T2-wheigted MRI though the anterior skull base of the index case. Absence of olfactory bulbs (blue arrows).

a. Hypo plastic left olfactory sulcus (white arrow).

b. Coronal section T2-wheigted MRI though the anterior skull base of a normal patient. Olfactory bulbs (white arrow). Taken from Carswell [6].

\section{Discussion}

The assessment of a hyposmia/anosmia should be carried out by an ENT specialist with a suitable material. Anamnesis should be focused in finding a history of craniofacial trauma, chronic nasal congestion or any other sign of obstructive nasal pathology. The antecedents of autoimmune or degenerative diseases such as Parkinson's, Alzheimer's and lupus erythematosus should also be assessed. ${ }^{4}$ Nasal endoscopy is mandatory in order to identify any structural alteration responsible for the symptoms. Septum deviation, polyps, adenoid hypertrophy and nasopharyngeal tumors should be ruled out. There are several tests to determine whether a person is able to smell or not. We used a simple and easy to perform smell test, the UPSIT, which showed the inability of our patient to perceive any odor at all.

The gold standard test to study the olfactory bulbs and tracks is the MRI. ${ }^{1,5}$ Any patient with anomia/hyposmia and no apparent structural or inflammatory disease should be studied with a MRI in order to rule out olfactory groove meningiomas, vascular anomalies or agenesis of the olfactory bulbs and tracts. ${ }^{2}$ The latter may be associated to other cerebral malformations, as in holoprosencephaly, where it is a constant finding, as well as being part of Kallmann syndrome, where the impossibility to smell is accompanied by a hypogonadotropic hypogonadism. Alterations in the olfactory bulbs development are 
also found in other genetic syndromes such as hemimegalencephaly and tuberous sclerosis complex. Our patient is one of the few cases reported in the medical literature of an isolated congenital agenesis of the olfactory bulbs ${ }^{1,5-7}$ with no other congenital abnormalities and a normal general development.

\section{Conclusion}

Olfactory alterations in the childhood and adolescence should not be treated as a trivial finding. Although usually underestimated, it should be studied carefully in order to identify a possible serious origin. It is necessary to asses all patients with a normal intranasal exploration with a thorough MRI evaluation of the olfactory bulbs and tracts, as well as identifying the potential associated abnormalities than an agenesis of these may imply, so that, if possible, an appropriate treatment could be given.

\section{Acknowledgements}

None.

\section{Conflicts of interest}

No potential conflicts of interest have to be disclaimed.

\section{Funding}

None.

\section{References}

1. Rienzo L, Artuso A, ColosimoC. Isolated Congenital Agenesis of the Olfactory Bulbs and Tracts in a Child without Kallmann's Syndrome. Annals of Otology, Rhinology \& Laryngology. 2002;111(7):657-660.

2. Sarnat HB. Olfactory bulb agenesis, hypoplasias and dysplasias, Canada. 2017.

3. Shetty S, Kapoor N, AmrithaJ, et al. Olfactory agenesis in Kallmann Syndrome (KS). J Clin Diagn Res. 2015;9(4):01.

4. Gaines . Anosmia and hyposmia. Allergy Asthma Proc. 2010;31(3):185-189.

5. Coimbra C, Cunha A, Ferreira E, et al. Isolated olfactory bulbs agenesis: An extremely rare entity Agenesia aislada de los bulbos olfativos: una entidad extremadamente rara. Acta Otolaringológica Española. 2016; 67(4):242-244.

6. Carswell A, Whinney D, Hollings N, et al. Isolated olfactory nerve agenesis. Bristish Journal of Hospital Medicine. 2008;69(8):474-475.

7. Zainnudin N, Razali F. Isolated congenital anosmia. Int J Otorhilaryngol Clin. 2016;8(2):82-86. 